\title{
Relationship between Ipsilateral Motor Deficits on the Less- Affected Side and Motor Function Stage on the Affected Side
}

\author{
Sung Min Son', Seok Hyun Nam², Kyung Woo Kang ${ }^{3}$, Dae Hyun Kim
}

1Department of Physical Therapy, College of Health Science, Cheongju University, Cheongju; 2Physical therapist, Respiratory Rehabilitation center, Yeungnam University Medical Center, Daegu; 3Department of Physical Therapy, Yeongnam University College, Daegu; 4Physical

therapist, Department of Physical Therapy, Chonbuk National Hospital, Jeonju, Korea

The Journal of Korean Physical Therapy

Volume $30 \bullet$ Number $6 \bullet$ December 2018 


\title{
Relationship between Ipsilateral Motor Deficits on the Less- Affected Side and Motor Function Stage on the Affected Side
}

\author{
Sung Min Son', Seok Hyun Nam², Kyung Woo Kang ${ }^{3}$, Dae Hyun Kim ${ }^{4}$ \\ 'Department of Physical Therapy, College of Health Science, Cheongju University, Cheongju; ${ }^{2}$ Physical therapist, Respiratory Rehabilitation center, \\ Yeungnam University Medical Center, Daegu; ${ }^{3}$ Department of Physical Therapy, Yeongnam University College, Daegu; ${ }^{4}$ Physical therapist, \\ Department of Physical Therapy, Chonbuk National Hospital, Jeonju, Korea
}

\begin{abstract}
Purpose: Aim of this study was to investigate whether there are ipsilateral motor deficits for visuospatial accuracy and fine movements by making a comparison between stroke patients and healthy subjects. We examined whether ipsilateral motor deficits are influenced by the level of functional movements and muscle strength of the upper and lower extremities of the affected side.

Methods: Thirty post-stroke subjects and 20 normal aged matched subjects were recruited. Outcome measures for less-affected side were the tracking task and nine-hole pegboard test. Fugl-Meyer test and motricity index were applied for the measurement of functional movements and muscle strength of affected side.

Results: Tracking task and nine-hole pegboard test was significantly different between control and experimental group. In terms of accuracy index according to tracking, the experimental group showed a lower accuracy index in the MCP joint than the control group. However, there were no significant difference relation between the level of motor function of the affected side and the motor deficit level of ipsilateral side.

Conclusion: Ipsilateral motor deficits may have significant clinical implications. It needs to be noted that although many patients, families, and medical staff are focused only on motor deficits of the affected side, motor deficits of the sound side can cause difficulties in daily living movements requiring delicate movements. In addition, there was no significant correlation between the level of motor function of the affected side and motor deficits of the sound side.
\end{abstract}

Keywords: Ipsilateral motor deficits, Less affected side, Stroke, Hemiparesis

\section{INTRODUCTION}

A representative neurological symptom resulting from stroke is hemiplegia involving motor and sensory impairments of the contralateral upper and lower extremities of the damaged cerebral hemisphere. ${ }^{1}$ Neuroanatomically, the cerebral hemisphere controls the motor function of the contralateral side of the body. Clinicians also perceived the ipsilateral upper and lower extremities of the impaired cerebral hemisphere as the sound side or the unaffected side and regarded them as the reference point for recovery of motor control of the affected side. ${ }^{2}$ Nevertheless, some nerve fibers are involved in conveying information for ipsilateral movements. Therefore, motor function may be affected not only in the contralateral extremity of

Received Nov 16, 2018 Revised Dec 23, 2018

Accepted Dec 26, 2018

Corresponding author Seok Hyun Nam

E-mail wearejjang@hanmail.net the cerebral hemisphere damaged by stroke but also in the ipsilateral extremity., Thus, the term "less affected side" is preferred to "unaffected side" in the area of rehabilitation science in recent. ${ }^{5-7}$

Liepmann ${ }^{8}$ reported that there was a problem with the expression of symbolic behavior in both upper extremities following unilateral brain injury and presented the ipsilateral motor deficit for the first time. Subsequently, Brodal reported that muscular weakness occurred in the ipsilateral extremity of the damaged cerebral hemisphere after unilateral brain injury, and this finding has been corroborated by many studies. ${ }^{5,6,10}$ Debaere et al. ${ }^{2}$ reported that interlimb coordination in the upper and lower extremities was reduced in patients with unilateral brain injury, compared with normal controls. In addition, it has been reported that there is a delay in reac-

Copylight (C2018 The Korea Society of Physical Therapy

This is an Open Access article distribute under the terms of the Creative Commons Attribution Non-commercial License (Http:// creativecommons.org/license/by-nc/4.o.) which permits unrestricted non-commercial use, distribution, and reproduction in any medium, provided the original work is properly cited. 
tion time and duration in the upper and lower extremities when goal-directed movements are performed. ${ }^{11}$ As described above, many previous studies have identified ipsilateral motor deficits such as functional movement deficits and delay of movement execution in hemiplegic patients than in healthy subjects. ${ }^{3,11,12}$

It has been reported that the ipsilateral motor deficits of hemiplegic patients is caused by the differences in the functional roles of two cerebral hemispheres. ${ }^{13-16}$ The ipsilateral descending pathway in the brain plays a role in the lateralization of motor control. ${ }^{4}$ The dominant hemisphere of the brain participates in the control of the upper extremities during complex tasks while the non-dominant hemisphere is responsible for the visuospatial accuracy required to perform tasks. ${ }^{3,4,12}$ Thus, the functional connectivity between the left and right cerebral hemispheres in the normal brain allows the successful implementation of goal-directed movements. However, patients with hemiplegia may experience ipsilateral motor deficits due to the anatomical damage to the neural circuits between the left and right hemispheres resulting from the damage to one cerebral hemisphere. In addition, Winstein et al. reported that if there is severe damage to the upper extremity of the paralysis side or there is damage in the internal capsule area of the brain, more severe ipsilateral motor deficits occur. ${ }^{17,18}$ However, the pathogenesis and factors affecting the development of ipsilateral motor deficits have not been clarified yet.

Therefore, the aim of this study was to investigate whether there are ipsilateral motor deficits for visuospatial accuracy and fine movements by making a comparison between stroke patients and healthy subjects, and to examine whether ipsilateral motor deficits are influenced by the level of functional movements and muscle strength of the upper and lower extremities of the affected side.

\section{METHODS}

\section{Subject}

In this study, the experimental group consisted of 20 patients diagnosed with stroke through evaluation of MRI or CT imaging findings of cerebral hemorrhage or cerebral infarction, and 20 healthy older people with no cognitive impairment, neurological impairment, or musculoskeletal disorders of arm and hand were included as controls. The inclusion criteria for stroke patients were as follows: (1) person diagnosed with unilateral cerebral infarction or cerebral hemorrhage, (2) person without cerebellar and brainstem damage, (3) person without visional problems such as unilateral neglect and hemianopsia, and (4) person who is judged to be able to understand and perform the tester's instructions on the basis of the MMSE-K (mini mental status examination Korean) score (24 points or higher).

In both the experimental and control groups, people with the right dominant hand were selected as the subjects, and the dominant side of the subjects was confirmed using the Edinburgh Handedness Inventor. All the participants understood the purpose of this study and voluntarily agreed to participate in the study before the experiment was conducted, and this study was conducted in accordance with the Declaration of Helsinki after obtaining the approval of the Institutional Review Board of Cheongju University.

\section{Measurement}

\section{1) Tracking task on metacarpal phalangeal joint}

In this study, the tracking task was performed to measure the dexterity of the metacarpal phalangeal joint. An electro-goniometer was used to measure the movements of the metacarpal phalangeal joint. The device consists of an electrical potentiometer with two hard arms connected to the springs, and it has been designed to allow the potentiometer to be placed on the same extension line of the second to fifth metacarpal phalangeal joint of the subject. In this tracking task, the metacarpophalangeal flexion of the second to fifth fingers in the sagittal plane is marked downward, but the metacarpophalangeal extension is marked upward, and the subject is required to track the target sine wave. During the tracking task, joint motion angles are measured and converted into digital signals by the electrogoniometer, and the data are transmitted to the personal computer. The transmitted signals are displayed on the computer screen using Lapview 8.5 (National Instrument, USA). In this experiment, to reduce the learning effect, the frequency and amplitude of the sine wave during practice trials were randomly varied within $\pm 20 \%$.

The subjects were instructed to track the red target sine wave on the computer screen as closely as possible for 15 seconds in a comfortable sitting position on the chair and the subjects' joint movements were indicated by white response sine waves. The accuracy index of the tracking task was calculated with the performance error value between the standard wave and the subject's performance. The subjects were fully informed about the experimental procedure before the tracking task, and testing was carried out after providing 
the subjects with the opportunity to perform two practice trials. The tracking task performance value in this study was the average of two measurements.

\section{2) Nine-hole pegboard test}

The nine-hole pegboard test was performed to measure the hand dexterity of the sound side. In this test, the subjects were instructed to remove nine pegs in the holes and reinsert them in the holes one at a time. The test was carried out by measuring the time from the moment the first peg was picked up to the moment the last peg was inserted in the hole again. The subjects were provided with sufficient explanation about the procedure. The test was conducted after the subjects were provided with the opportunity to perform two practice trials. The mean of the two measurements in this study was used for analysis.

\section{3) Fugl-Meyer test}

Fugl-Meyer test was used to evaluate the function of upper and lower extremities of the affected side in stroke patients. This test is composed of 50 items based on the Brunnststrom hemiplegia classification and six stages of stroke recovery, and is used as a qualitative test method for testing motor performance after stroke. The total score is 100 points, which consists of 66 points for upper limb function and 33 points for lower limb function.

\section{4) Motricity index}

The Motricity index was used to evaluate the degree of muscle strength of the upper and lower extremities in stroke subjects. This test is used to assess shoulder abduction, elbow flexion, pinch grip in the upper limb and hip flexion, knee extension, and ankle joint dorsiflexion in the lower limb. The score of this assessment ranges from 0 to 99 points for each of the upper and lower extremities. The reliability and validity of the Motricity Index was verified by Collin and Wade's study. ${ }^{19}$

\section{Statistical analysis}

An independent t-test was used to compare the age and perfor- mance of tracking task and nine-hole pegboard test of the two groups. In addition, Pearson correlation coefficient was used to eaxmine the correlation between the function of upper and lower extremities of the affected side (Fugl-Meyer test and Motricity index) and ipsilateral motor deficits. The collected data were analyzed using SPSS ver. 22.0 (IBM Co., Armonk, NY, USA). The level of statistical significance was set at $\mathrm{p}<0.05$.

\section{RESULTS}

Two group demographic data are summarized in Table 1. No significant difference was found in age and gender between control and experimental group. The means \pm SD of the tracking task and nine-hole pegboard test time of both group are shown in Table 2. Tracking task and nine-hole pegboard test were significantly different between control and experimental group. In terms of accuracy index according to tracking, the experimental group showed a lower accuracy index in the MCP joint than the control group (Table 3). A more time of nine-hole pegboard test in relation to hand function was observed in the stroke group, compared to the control group. However, in the analysis of the relation between the level of motor

Table 1. The general characteristics of subjects

\begin{tabular}{lcc}
\hline & $\begin{array}{c}\text { Control group } \\
(\mathrm{n}=30)\end{array}$ & $\begin{array}{c}\text { Experimental group } \\
(\mathrm{n}=30)\end{array}$ \\
\hline Gender (Male/Female) & $10 / 20$ & $10 / 20$ \\
Age (year) & $57.55 \pm 9.23$ & $54.33 \pm 9.35$ \\
Onset duration (month) & - & $22.80 \pm 12.72$ \\
Stroke Type & - & $15 / 15$ \\
(Hemorrhage/Infarction) & - & $11 / 19$ \\
Paretic side (Right/Left) & - & $23.73 \pm 3.87$ \\
Fulg-meyer test & - & $19.73 \pm 1.54$ \\
UE & - & \\
LE & - & $59.30 \pm 3.23$ \\
Motircity index & - & \\
UE & - & \\
LE & & \\
\hline
\end{tabular}

Mean \pm S.D

UE: upper extremity, LE: lower extremity.

Table 2. Comparison of tracking task and nine-hole pegboard test in the experimental and control group

\begin{tabular}{|c|c|c|c|c|}
\hline Parameters & Control group $(n=30)$ & Experimental group $(n=30)$ & $\mathrm{t}$ & p-value \\
\hline Tracking task (RMS error, volt) & $13.77 \pm 5.33$ & $10.42 \pm 5.21$ & 2.21 & $0.035^{*}$ \\
\hline Nine-hole pegboard test (second) & $23.48 \pm 3.21$ & $25.42 \pm 3.16$ & 2.12 & $0.039^{*}$ \\
\hline
\end{tabular}

${ }^{*}$ significant difference between experimental and control group $\left({ }^{*} p<0.05\right)$.

RMS: root mean square. 
Table 3. Correlation between tracking task, nine-hole pegboard test, Fulg-Meyer test, and Motriciy index

\begin{tabular}{|c|c|c|c|c|c|c|c|c|c|c|}
\hline & \multicolumn{2}{|c|}{ Tracking test } & \multicolumn{2}{|c|}{$\mathrm{NHP}$} & \multicolumn{2}{|c|}{ FMT-UE } & \multicolumn{2}{|c|}{ FMT-LE } & \multicolumn{2}{|c|}{ MI-UE } \\
\hline & $r$ & $\mathrm{p}$ & $r$ & $p$ & $r$ & $\mathrm{p}$ & $r$ & $\mathrm{p}$ & $r$ & $p$ \\
\hline Tracking test & 1 & & & & & & & & & \\
\hline $\mathrm{NHP}$ & -0.746 & 0.000 & 1 & & & & & & & \\
\hline FMT-UE & 0.054 & 0.777 & 0.45 & 0.812 & 1 & & & & & \\
\hline FMT-LE & 0.253 & 0.117 & 0.005 & 0.415 & 0.774 & 0.000 & 1 & & & \\
\hline MI-UE & 0.117 & 0.537 & 0.005 & 0.98 & 0.884 & 0.000 & 0.729 & 0.000 & 1 & \\
\hline MI-LE & 0.292 & 0.118 & -0.117 & 0.538 & 0.843 & 0.000 & 0.827 & 0.000 & 0.819 & 0.000 \\
\hline
\end{tabular}

NHP: nine-hole pegboard test, FMT: Fulg-Meyer test, MI: Motircity index, UE: upper extremity, LE: lower extremity.

function (Fugl-Meyer test, Motricity index) of the affected side and the motor deficit level of ipsilateral side, no significant relation was found.

\section{DISCUSSION}

The present study examined the presence of ipsilateral motor deficits in stroke patients by comparing visuospatial accuracy and fine movements between stroke patients and normal controls. In the experimental group, the sound side was the right side in 19 patients and the left side in 11 patients. Therefore, in the control group, the tracking task and the pegboard nine-hole test were performed with the right hand in 19 subjects and with the left hand in 11 subjects. Regarding the results of this study, the accuracy index of the tracking task and the results of the pegboard nine-hole test to assess hand dexterity revealed reduced hand function in the experimental group, compared to the control group. In addition, we examined the correlation between the level of ipsilateral motor deficits and the levels of functional movement and muscle strength of the upper and lower extremities of the affected side. The results of this study showed that the functional level and the degree of muscle strength of the upper extremity of the affected side were not correlated with ipsilateral motor deficit, which means that ipsilateral motor deficit is not related to the level of functional impairment of the upper extremity of the affected side.

In the present study, reduced hand function of the sound side was observed in the experimental group of stroke patients, compared with the control group, and this result suggests that chronic stroke patients also have ipsilateral motor deficits of the unaffected side. When movements with a high level of task complexity and difficulty are performed, both the cerebral hemispheres are involved simultaneously. ${ }^{13}$ fMRI study showed that not only the activation of the contralateral hemisphere but also the activation of the ipsilateral hemisphere occurs when a unilateral complex task is performed. ${ }^{16}$ In addition, they noted that the activation of both hemispheres increased as the task difficulty increased. ${ }^{18,20}$ The tracking task is visuospatial movement that requires eye-hand coordination and closed looped movement that requires visual feedback. ${ }^{21}$ The ninehole pegboard test is a brief, standardized, quantitative test of upper extremity function, and is used to measure complex continuous movements that require precision and dexterity. Therefore, the tracking task and the nine-hole pegboard test performed in the present study are thought to be tasks requiring mobilization of both the left and right cerebral hemispheres. In this way, to perform complex movements, such as goal-directed target movements, both hemispheres are mobilized together. It is known that unilateral brain injury is impairment of the central processing ability of higher-level cognitive functions, and it could induce ipsilateral motor deficits. ${ }^{11,15}$ Several previous studies have reported that there are various types of ipsilateral motor deficits such as increased segmentation of movement, inaccuracy of visuospatial movements, and temporal inconsistency in the upper limb of the sound side during repetitive movements after stroke, ${ }^{10,11,22,23}$ and these findings are consistent with the results of this study.

In addition, the results of this study showed no significant correlations between the levels of functional movements and muscle strength of the affected side and ipsilateral motor deficits. A previous study of the effects of bilateral upper limb training on motor deficits of the sound side reported that bilateral upper limb training of stroke patients is effective for motor deficits of the sound side, and that there is no correlation between the recovery of ipsilateral motor deficits and the functional level of the affected side. These results are similar to those of the present study which showed that there was no significant difference between the degree of ipsilateral motor deficits 
and the level of functional movements of the affected side. ${ }^{24}$ In addition, Winstein et al. and Shibasaki et al. reported that the degree of motor deficits in the ipsilateral upper extremity is more severe as the level of task difficulty is higher. ${ }^{18,20}$ Thus, the results of this study suggest that ipsilateral motor deficits are not influenced by the degree of damage of the paralysis side and they are more affected by the level of task difficulty.

In this study, it was found that there were significant motor deficits related to hand accuracy and exercise dexterity in both the affected and sound sides after stroke. Ipsilateral motor deficits may have significant clinical implications. It needs to be noted that although many patients, families, and medical staff are focused only on motor deficits of the affected side, motor deficits of the sound side can cause difficulties in daily living movements requiring delicate movements. In addition, there was no significant correlation between the level of motor function of the affected side and motor deficits of the sound side. However, there are some limitations to consider in terms of interpretation of the results of the present study. First, the sample size was not large enough to determine the correlation. Second, the rates of females and males and the rates of paralysis sides (left and right) were not equal. In future studies, these limitations need to be supplemented, and there is a need for further research on the effects of items such as the paralysis side, duration of disease and type of stroke.

\section{ACKNOWLEDGEMENTS}

This work was supported by the National Research Foundation of Korea (NRF) grant funded by the Korea government (MSIT)

(NRF-2017R1C1B5017702)

\section{REFERENCES}

1. Kwakkel G, Kollen BJ, van der Grond J et al. Probability of regaining dexterity in the flaccid upper limb: impact of severity of paresis and time since onset in acute stroke. Stroke. 2003;34(9):2181-6.

2. Debaere F, Van Assche D, Kiekens C et al. Coordination of upper and lower limb segments: deficits on the ipsilesional side after unilateral stroke. Exp Brain Res. 2001;141(4):519-29.

3. Kwon YH, Kim CS, Jang SH. Ipsi-lesional motor deficits in hemiparetic patients with stroke. NeuroRehabilitation. 2007;22(4):279-86.

4. Schaefer SY, Haaland KY, Sainburg RL. Hemispheric specialization and functional impact of ipsilesional deficits in movement coordination and accuracy. Neuropsychologia. 2009;47(13):2953-66.

5. Alagona G, Delvaux V, Gerard P et al. Ipsilateral motor responses to focal transcranial magnetic stimulation in healthy subjects and acutestroke patients. Stroke. 2001;32(6):1304-9.

6. Chestnut C, Haaland KY. Functional significance of ipsilesional motor deficits after unilateral stroke. Arch Phys Med Rehabil. 2008;89(1):62-8.

7. Sunderland A. Recovery of ipsilateral dexterity after stroke. Stroke. 2000; 31(2):430-3.

8. Liepmann H, Mass O. Fall von linksseitiger agraphie und apraxie bei rechsseltiger lahmung. Journal für Pyschologie und Neurologie. 1907.

9. Brodal A. Self-observations and neuro-anatomical considerations after a stroke. Brain. 1973;96(4):675-94.

10. Esparza DY, Archambault PS, Winstein CJ et al. Hemispheric specialization in the co-ordination of arm and trunk movements during pointing in patients with unilateral brain damage. Exp Brain Res. 2003;148(4): 488-97.

11. Kim SH, Pohl PS, Luchies CW et al. Ipsilateral deficits of targeted movements after stroke. Arch Phys Med Rehabil. 2003;84(5):719-24.

12. Hermsdorfer J, Ulrich S, Marquardt C et al. Prehension with the ipsilesional hand after unilateral brain damage. Cortex. 1999;35(2):139-61.

13. Catalan MJ, Honda M, Weeks RA et al. The functional neuroanatomy of simple and complex sequential finger movements: a pet study. Brain. 1998;121(Pt 2):253-64.

14. Lotze M, Scheler G, Tan HR et al. The musician's brain: functional imaging of amateurs and professionals during performance and imagery. Neuroimage. 2003;20(3):1817-29.

15. Pujol J, Roset-Llobet J, Rosines-Cubells D et al. Brain cortical activation during guitar-induced hand dystonia studied by functional MRI. Neuroimage. 2000;12(3):257-67.

16. Sadato N, Campbell G, Ibanez V et al. Complexity affects regional cerebral blood flow change during sequential finger movements. J Neurosci. 1996;16(8):2691-700.

17. Darling WG, Pizzimenti MA, Hynes SM et al. Volumetric effects of motor cortex injury on recovery of ipsilesional dexterous movements. Exp Neurol. 2011;231(1):56-71.

18. Winstein CJ, Pohl PS. Effects of unilateral brain damage on the control of goal-directed hand movements. Exp Brain Res. 1995;105(1):163-74.

19. Collin C, Wade D. Assessing motor impairment after stroke: a pilot reliability study. J Neurol Neurosurg Psychiatry. 1990;53(7):576-9.

20. Shibasaki H, Sadato N, Lyshkow H et al. Both primary motor cortex and supplementary motor area play an important role in complex finger movement. Brain. 1993;116 (Pt 6):1387-98.

21. Harvey M, Milner AD, Roberts RC. Spatial bias in visually-guided reaching and bisection following right cerebral stroke. Cortex. 1994; 30(2):343-50.

22. Kim CS, Kwon YH, Kim K. Ipsilateral motor deficit in patients with unilateral brain damage. J Kor Soc Phys Ther. 2006;18(4):1-9.

23. Desrosiers J, Bourbonnais D, Bravo G et al. Performance of the 'unaffected' upper extremity of elderly stroke patients. Stroke. 1996;27(9): 1564-70.

24. Kaeser M, Wyss AF, Bashir S et al. Effects of unilateral motor cortex lesion on ipsilesional hand's reach and grasp performance in monkeys: relationship with recovery in the contralesional hand. J Neurophysiol. 2010;103(3):1630-45. 